\title{
A IMPLANTAÇÃO DE UM NÚCLEO DE CAPACITAÇÃO DOCENTE NA UNIVERSIDADE FEDERAL DE JUIZ DE FORA
}

DOI: 10.37702/2175-957X.COBENGE.2021.3491

Cristiano Gomes Casagrande - cristiano.casagrande@ufjf.edu.br Universidade Federal de Juiz de Fora

Rua Subtenente Omar Pereira 231

36047-190 - Juiz de Fora - MG

Danilo Pereira Pinto - danilo.pinto@ufjf.edu.br

Universidade Federal de Juiz de Fora

Rua Antônio Maria de Souza 147

36025-310 - Juiz de Fora - MG

Roberta Cavalcanti Pereira Nunes - rcpnunes@gmail.com

Universidade Federal de Juiz de Fora

Rua Oscavo Gonzaga Prata 372

36033-220 - Juiz de Fora - MG

Taís Borges Oliveira - tais.borges@engenharia.ufjf.br

UFJF

Avenida Presidente Itamar Franco 2565

36025-290 - Juiz de Fora - MG

Resumo: As novas Diretrizes Curriculares Nacionais para o Curso de Engenharia (DCN 2019) prescrevem a obrigatoriedade de que as instituições de ensino superior (IES) mantenham programas contínuos de capacitação docente. Sendo assim, foi criado na Universidade Federal de Juiz de Fora (UFJF) o Núcleo FOCO Núcleo de Formação Continuada dos Docentes da Faculdade de Engenharia, que tem como um dos seus objetivos construir um programa de capacitação continuada docente para os cursos de Engenharia da instituição, capaz de atender à legislação vigente e propor a qualificação permanente dos docentes, tornando a atividade docente um processo efetivo de ensino aprendizagem. O presente trabalho apresenta os objetivos, a metodologia e as ações iniciais desse Núcleo, destacando os principais resultados obtidos até o momento.

Palavras-chave: capacitação docente, formação continuada, Diretrizes 


\section{(C. COBENGE e IV Simpósio Internacional de Educação em Engenharia

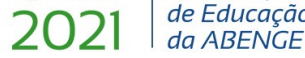 28 a 30 de SETEMBRO

Curriculares Nacionais 


\section{A IMPLANTAÇÃO DE UM NÚCLEO DE CAPACITAÇÃO DOCENTE NA UNIVERSIDADE FEDERAL DE JUIZ DE FORA}

\section{INTRODUÇÃO}

Os cursos de Engenharia vêm enfrentando o desafio de construir um novo modelo de ensino aprendizagem capaz de atender as atuais demandas da sociedade. A reestruturação produtiva e a criação de novas relações econômicas e de trabalho, bem como o processo de intensificação de incorporação de novas tecnologias à produção, exigem que os novos engenheiros dominem um conjunto amplo de conceitos e informações e que exerçam o seu trabalho de forma cada vez mais multidisciplinar. A aplicação de muitas dessas novas tecnologias vem causando impactos sociais e ambientais cada vez mais abrangentes e complexos, o que, obrigatoriamente, deve ser levado em conta nos projetos de engenharia. Adicionalmente, verifica-se uma crescente ampliação do campo de atividade dos engenheiros para áreas gerenciais e administrativas.

Essa realidade passa a demandar um profissional de engenharia crítico, empreendedor, criativo e capaz de dar respostas adequadas aos novos problemas que resultam de uma dinâmica de transformações que vem ocorrendo de forma intensa e profunda em todos os setores (PINTO et al, 1999).

A formação em Engenharia no Brasil experimentou, recentemente, dois momentos importantes: em 09 de abril de 2002, com a Resolução MEC/CNE/CES n 11/2002 (MEC/CNE/CES, 2002), que trata das Diretrizes Curriculares Nacionais para os cursos de Engenharia (DCN 2002); e em 26 de abril de 2019, com a publicação no Diário Oficial da União (DOU) da Resolução MEC/CNE/CES 02/2019 (MEC/CNE/CES, 2019), com as novas Diretrizes Curriculares Nacionais do Curso de Graduação em Engenharia (DCN 2019).

Observando o panorama anterior às DCN 2002 e 2019, percebe-se, em ambos os casos, elevadas taxas de evasão e retenção nos cursos de Engenharia, principalmente nos dois primeiros anos, e um contexto no qual o mundo do trabalho exige um profissional mais completo, não exclusivamente com as competências técnicas, mas também com uma formação mais humanística e empreendedora. Portanto, as premissas colocadas para a reformulação das DCN em 2002 e 2019 são idênticas: busca pela qualidade na formação, flexibilizar os currículos dos cursos e reduzir a evasão.

Em 2002, pretendia-se motivar os alunos para as engenharias, desenvolver novas concepções curriculares, com novas metodologias de ensino aprendizagem e processos avaliativos, que resultassem em autonomia intelectual para os alunos. Após 2002, houve o crescimento do número de cursos e vagas e um grande aumento do número de cursos de educação à distância (EaD), porém, com elevada taxa de ociosidade de vagas. As taxas de evasão e retenção continuaram elevadas, o que leva a crer que as mudanças estabelecidas nas DCN 2002 não foram implementadas de forma efetiva, portanto, não promovendo transformações no ensino de Engenharia no Brasil.

As DCN 2019 abrem a perspectiva de um novo entendimento sobre a flexibilidade dos cursos, a importância e a construção coletiva do Projeto Pedagógico do Curso (PPC), além da capacitação dos docentes para implementação de metodologias de aprendizagem ativa, tornando o aluno o centro deste processo. Reforçam também a obrigatoriedade da adoção de sistema de acolhimento e nivelamento, visando a redução dos índices de retenção e evasão. Propõe-se que sejam definidos currículos a partir de competências a serem desenvolvidas, ao invés de núcleos de conteúdo. Dessa forma, os conteúdos serão 
implementados dentro de contextos de desenvolvimento de competências e contextualizados em ambientes de engenharia (PINTO e CASAGRANDE, 2020).

Com relação ao corpo docente, as DCN 2019 incluem a obrigatoriedade das Instituições de Ensino Superior (IES) manterem um programa permanente de formação e desenvolvimento do mesmo, com vistas à valorização da atividade de ensino, ao maior envolvimento dos professores com o PPC e ao aprimoramento em relação à proposta formativa.

Observa-se que alguns docentes da Faculdade de Engenharia da Universidade Federal de Juiz de Fora (FACENG/UFJF) demonstram grande interesse pela área de Educação em Engenharia. Desenvolvem pesquisa, produzem artigos técnicos e têm participação efetiva ao longo dos anos no Congresso Brasileiro de Educação em Engenharia (COBENGE).

Os Encontros de Educação em Engenharia (EEE), realizados de 1995 a 2005, em parceria entre a Universidade Federal de Juiz de Fora (UFJF) e a Universidade Federal do Rio de Janeiro (UFRJ) (PINTO e OLIVEIRA, 2007), buscavam proporcionar o debate sobre questões relativas à formação de engenheiros, ao aprimoramento das técnicas de ensino e aprendizagem e à integração acadêmica entre docentes e alunos das instituições promotoras e participantes. Essa parceria resultou em duas tentativas de criação de programas de pós-graduação com cursos de Mestrado e Doutorado na área de Educação em Engenharia - Programa Cooperativo I (1999) e II (2005) (PINTO et al, 1999).

Antes mesmo da publicação das DCN 2019, a FACENG/UFJF tentou implementar ações com o objetivo de analisar as DCN e propor a elaboração dos novos PPC dos cursos de Engenharia ofertados, sendo inclusive criado um fórum permanente de Educação em Engenharia para isso. Entretanto, suas atividades não foram expressivas e não tiveram continuidade. A Direção também propôs a criação de grupos de estudo voltados para temáticas como extensão, metodologias ativas e formação por competências, mas que não chegaram a ser implementados. Concomitantemente, uma outra proposta, originada nos grupos dos Programas de Educação Tutorial da Engenharia, foi a criação de um grupo de estudos em metodologias de aprendizagem ativa, aberto à participação de todos os docentes da FACENG/UFJF. Esta proposta acabou também por não se concretizar (PINTO e CASAGRANDE, 2020).

Essas iniciativas frustradas demonstram claramente uma dificuldade de implantar ações institucionalizadas de capacitação docente e formação continuada em educação para Engenharia, mesmo no momento em que se discutia as DCN. Entretanto, amparado nas obrigatoriedades de capacitação continuada de docentes imposta pelas DCN 2019 e no grande desafio da construção dos Projetos Pedagógicos de Cursos (PPC), baseados em competências e com todas as características colocadas nas DCN, os autores deste trabalho identificaram que era o momento propício para elaborar uma proposta de criação de um programa de capacitação, a ser construído coletivamente pelos docentes.

\section{A CRIAÇÃO DO NÚCLEO FOCO}

Atualmente, na FACENG/UFJF, identificam-se ações pontuais de alguns docentes no sentido de utilizarem metodologias ativas de ensino aprendizagem. Todavia, não se pode dizer que há na instituição o uso amplo e sistematizado de metodologias de aprendizagem ativa nos cursos (PINTO e CASAGRANDE, 2020).

Além disso, não havia nenhuma ação estruturada de capacitação dos docentes. $O$ ingresso na carreira não exige nenhuma prática docente, bastando o título de doutor e aprovação em processo seletivo. Ao iniciar a carreira, ao docente são atribuídas atividades 
acadêmicas, mesmo sem a adequada capacitação em docência. Supõe-se que, ao ser selecionado, este está apto a desenvolver atividades acadêmicas, com grupos de alunos, e atividades administrativas. Durante o estágio probatório, o docente tem uma oportunidade de capacitação, através da Pró-Reitoria de Recursos Humanos, no entanto, muitos a consideram apenas uma obrigatoriedade.

Identificada a carência de um programa de capacitação docente na FACENG/UFJF, que atendesse a legislação vigente, buscando valorizar a prática docente e que fosse perene, foi proposta a criação do Núcleo de Formação Continuada dos Docentes da Faculdade de Engenharia, o Núcleo FOCO.

Os objetivos desse Núcleo são:

- Estabelecer um programa de capacitação para desenvolver os saberes docentes e sua mobilização dentro da sala de aula;

- Qualificar permanentemente docentes que atuam nos cursos de Engenharia da UFJF;

- Tornar a atividade docente um processo efetivo de ensino aprendizagem.

Como resultados das ações do Núcleo, espera-se:

- Atender a legislação da educação superior: DCN das Engenharias;

- Melhorar a qualidade da formação dos egressos;

- Aumentar a satisfação/entusiasmos/dedicação/motivação na prática docente;

- Promover a integração entre docentes;

- Auxiliar no aprimoramento dos PPCs;

- Melhorar os indicadores das avaliações de cursos realizadas pelo Instituto Nacional de Estudos e Pesquisas Educacionais Anísio Teixeira (INEP).

\section{RESULTADOS}

A proposta de criação de um programa de capacitação docente foi apresentada ao Conselho de Unidade da FACENG/UFJF e aprovada em 15 de julho de 2020. Através da Portaria/SEI $n^{\circ} 04$ de 26 de janeiro de 2021, foi criado e institucionalizado o Núcleo FOCO e sua primeira Comissão Coordenadora.

Foram realizadas reuniões com parceiros estratégicos da UFJF para apresentação da proposta do Núcleo e desenvolvimento de contatos de apoio entre as instâncias, entre elas, o ICE - Instituto de Ciências Exatas, a Pró-Reitoria de Extensão e a Pró-Reitoria de Graduação, todas receptivas e de acordo com a parceria.

Em novembro e dezembro de 2020, foi realizada uma pesquisa de Diagnóstico da Prática Docente da FACENG/UFJF, na qual se procurou identificar o perfil dos docentes (departamento, ano de ingresso na carreira, capacitação em docência e metodologias de ensino aprendizagem, participação em eventos de capacitação em docência), as práticas de ensino, as metodologias utilizadas em sala de aula e o interesse pela formação continuada.

Houve uma expressiva participação dos docentes $(78,42 \%)$, totalizando 109 respostas ao questionário. Entre as respostas, pode-se perceber que:

1. Há uma baixa capacitação formal em práticas de ensino e aprendizagem por parte dos docentes, mas ao mesmo tempo, um grande interesse no assunto; 
2. Apesar de predominarem aulas ministradas de forma tradicional (aulas expositivas com avaliações de conteúdos), os docentes já utilizam várias estratégias de metodologias de aprendizagem ativa, muitas vezes de maneira não intencional;

3. Há dúvidas sobre o tema metodologias de aprendizagem ativa e aplicação dessas estratégias, observado pela alta taxa de respostas intermediárias ("não tenho certeza se faço uso" e indiferença ao analisar as afirmações a respeito).

Os resultados reforçaram a importância da criação, por parte da Direção da FACENG/UFJF, do Núcleo FOCO, visando apoiar iniciativas de práticas inovadoras em sala de aula, aprimorando o ensino de engenharia de acordo com a legislação vigente.

Através das respostas ao diagnóstico, foi possível identificar um conjunto de temas sugeridos para capacitação. A Tabela 1 apresenta a lista de temas propostos.

Tabela 1 - Lista de Temas para Capacitação Docente.

$$
\text { Temas }
$$

Formação/ensino por competências

Avaliação por competências, currículo por competências

Saberes docentes, docência no ensino superior

Prática na Educação em Engenharia; como aprendemos (pirâmides de Glaser, taxonomia de Bloom etc.); soft skills na educação superior

\begin{tabular}{c}
\hline Percurso formativo \\
\hline Neuro educação \\
\hline PPC - Construção do Projeto Pedagógico de Curso \\
\hline Oegislações: DCN, relatórios da UNESCO sobre a educação, etc. \\
O cenário atual da educação \\
Troca de experiências sobre práticas docentes \\
\hline Métodos de avaliação \\
\hline Atividades extracurriculares \\
\hline Motivação/engajamento de alunos \\
\hline Ensino remoto \\
\hline Universidade empreendedora
\end{tabular}

Fonte: Os autores

A partir da institucionalização do Núcleo FOCO, foi desenvolvido um folder eletrônico (Figura 1) para auxiliar na divulgação, e o site <https://www.ufjf.br/nucleofoco/> com o objetivo de divulgar as ações do núcleo, disponibilizar referências, síntese das atividades realizadas, documentos, etc.

Outra ação do Núcleo FOCO foi iniciar a criação de grupos de estudos. Esses grupos deverão funcionar de maneira independente, preferencialmente com a participação de docentes e TAEs - Técnicos Administrativos em Educação - de todos os departamentos da Faculdade de Engenharia.

Os grupos de estudos têm como objetivo apresentar propostas de sugestões que serão encaminhadas para discussão nos departamentos e posterior análise pelo Conselho de Unidade. Os grupos realizarão estudos de práticas atuais, legislação e temas como mostrados na Tabela 1, dentre outros, e, posteriormente, irão identificar possíveis medidas que visem a valorização da profissão do professor. Como propostas inicias de estudo foram criados os seguintes grupos: 
Figura 1 - Folder de Apresentação do Núcleo FOCO/UFJF.

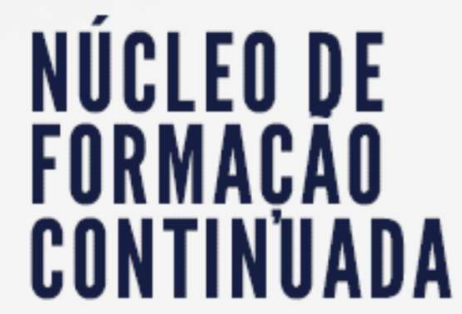

QUEM SOMOS?
DOS DOCENTES

DA FACULDADE

DE ENGENHARIA UMA CONSTRUÇÃO COLETIVA DE UM
PROGRAMA PERMANENTE DE CAPACITAÇÃO
DOCENTE

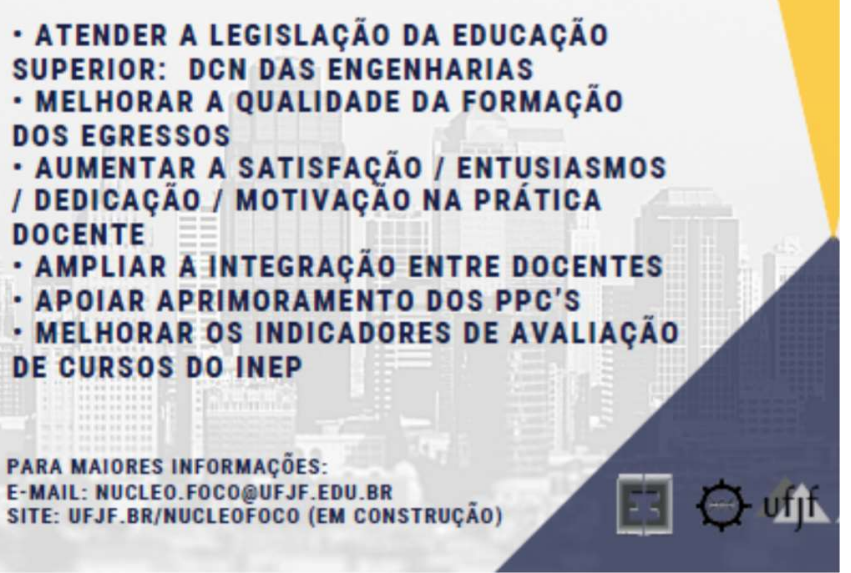

Fonte: Os autores.

- Capacitação inicial durante estágio probatório:

O período do estágio probatório é uma oportunidade de capacitar os novos docentes. O ingresso na carreira está, quase sempre, condicionado à obtenção do título de doutor e à aprovação em processo seletivo. Muitos novos docentes ingressam na carreira sem nenhuma prática. Em geral, as Instituições de Ensino Superior (IES) não oferecem capacitação para a docência na chegada de novos membros ou, quando oferecem, esta não é considerada adequada.

Este grupo de estudos pretende analisar os cursos ofertados pela UFJF, identificar possibilidades de complementação desses cursos ofertados, propor critérios de valorização da capacitação docente durante o estágio probatório e outras medidas que auxiliem os novos docentes a vencer as dificuldades iniciais (acolhimento, administrativas, pedagógicas, dentre outras).

- Valorização da Capacitação Docente Continuada em Engenharia:

Atualmente há pouca ou nenhuma valorização nas atividades de docência para os cursos de graduação. Apenas há a obrigatoriedade de ministrar 8 (oito) horas de aulas semanais na graduação. Na maior parte das vezes, os critérios apreciados para evolução na carreira do magistério superior concentram-se, principalmente, na pesquisa, seguidos das atividades de extensão e pós-graduação. 
Assim, sem os devidos incentivos, o estudo e o aprimoramento da prática docente tornam-se objetivos secundários ao longo da carreira desses servidores. O objetivo deste grupo é analisar e criar propostas de novos critérios avaliativos formais e institucionais que valorizem as atividades da docência nos cursos de graduação em Engenharia e a capacitação contínua nessas temáticas.

- Programa de Suporte e Acompanhamento da Prática Docente:

Os docentes com a carreira já consolidada muitas vezes se deparam em algum momento com uma falta de interesse na docência ou com dificuldades de renovação das suas práticas de ensino. Para esses casos, a ideia de formação de um programa para trabalhar essas questões e a possibilidade de fazer isso em conjunto com outros docentes vem a ser uma alternativa pertinente.

Este grupo de estudos tem como objetivo propor um programa cíclico que possa acolher os docentes interessados em um grupo para acompanhamento, suporte e trocas para análise, reflexão e melhoria da sua própria prática docente. Este programa poderá abranger metodologias de avaliação (docentes e discentes), metodologias de ensino aprendizagem, valorização dos pontos fortes do docente em sala de aula, identificação de pontos de melhoria nas atividades de sala de aula, dentre outros.

Os grupos de estudos trabalharão por aproximadamente 5 (cinco) meses, e entregarão ao final um relatório propositivo que abranja os objetivos acima citados. Pretende-se criar posteriormente outros grupos de estudos em temáticas demandadas pelos docentes da FACENG/UFJF, como elaboração do PPC, formação por competências, aprendizagem ativa, dentre outros.

\section{CONCLUSÕES}

Conforme visto, o Núcleo FOCO foi desenvolvido com o objetivo de atender as DCN 2019 no que tange à capacitação docente, e obteve apoio da Direção e dos docentes da FACENG/UFJF desde o momento de sua idealização e criação. O diagnóstico da prática docente, a primeira ação do Núcleo, teve uma participação muito efetiva dos docentes, como apresentado anteriormente.

A criação dos grupos de estudos foi outra atividade marcante proporcionada pelo Núcleo. Eles tiveram a adesão inicial de 23 docentes e 3 Técnicos-Administrativos em Educação, e representando $87,5 \%$ dos departamentos da FACENG/UFJF.

Foram identificados alguns desafios que precisarão ser contornados. O principal deles está associado à motivação e engajamento dos docentes em participar das atividades. Isso se tornou claro visto que, ao mesmo tempo em que a adesão ao formulário de diagnóstico tenha sido alta e os resultados tenham confirmado o desejo e a necessidade em se trabalhar com a temática da capacitação docente, a participação efetiva nas reuniões e eventos promovidos até agora pelo Núcleo tem sido baixa.

O trabalho do Núcleo para os próximos meses estará voltado para estimular o engajamento dos docentes, para a continuidade da promoção de eventos de discussão acerca das temáticas e necessidades identificadas, e para a continuidade dos trabalhos dos grupos de estudos. O Núcleo FOCO também está buscando conseguir bolsistas para aprimorar suas capacidades de entrega e prevê-se um foco maior na temática dos PPCs de cursos para os próximos meses, em decorrência dos prazos de revisão e elaboração estabelecidos legalmente. 


\title{
REFERÊNCIAS
}

MEC/CNE/CES. Resolução CNE/CES 11 de março de 2002: Diretrizes Curriculares Nacionais do Curso de Graduação em Engenharia. Diário Oficial da União, Brasília, 09 de abril de 2002, seção 1, p. 32.

MEC/CNE/CES. Resolução CNE/CES 2 de 24 de abril de 2019: Diretrizes Curriculares Nacionais do Curso de Graduação em Engenharia. Diário Oficial da União, Brasília, 26 de abril de 2019, seção 1, p. 43.

OLIVEIRA, Vanderli Fava de; PINTO, Danilo Pereira. Educação em Engenharia como Área do Conhecimento. In: PINTO, Danilo Pereira; NUNES, Roberta C. Pereira; OLIVEIRA, Vanderli Fava de (Org.). Educação em Engenharia: Evolução, Bases e Formação. 1 ed. Juiz de Fora: Fórum Mineiro de Engenharia de Produção, 2010, p. 91106.

PINTO, Danilo Pereira et al. Educação em Engenharia: a necessidade de um Programa de Mestrado e Doutorado. In: XXVII Congresso Brasileiro de Ensino de Engenharia, 1999, Natal/RN. Anais. Natal/RN.

PINTO, Danilo Pereira; CASAGRANDE, Cristiano Gomes. Diretrizes Curriculares Nacionais: Comentários Gerais e Acompanhamento da Implantação na Universidade Federal de Juiz de Fora. In: XLVIII Congresso Brasileiro de Ensino de Engenharia, 2020, Evento online. Anais.

PINTO, Danilo Pereira; OLIVEIRA, Vanderli Fava de. Engineering Education as an Area of the Scientific Knowledge. In: International Conference on Engineering Education, 2007, Coimbra. Anais do ICEE 2007. Coimbra, Portugal.

\section{IMPLEMENTATION OF A TEACHING QUALIFICATION CENTER AT THE FEDERAL UNIVERSITY OF JUIZ DE FORA}

\begin{abstract}
Brazilian Curriculum Guidelines for Engineering Course (DCN 2019) prescribe the requirement to colleges maintain continuous programs of teaching qualification for professors. Therefore, it was created the FOCO Center - Center of Continuing Education of School of Engineering, at the Federal University of Juiz de Fora. FOCO Center has as one of its objectives to build a program of continuing qualification for professors for Engineering courses at college, capable of complying with current legislation and proposing the permanent qualification for professors, making teaching activity an effective teachinglearning process. This paper presents objectives, methodology and initial actions of this Center, emphasizing the main results obtained so far.
\end{abstract}

Keywords: teaching qualification, continuing education, Brazilian Curriculum Guidelines. 